\title{
Trichostatin A increases radiosensitization of tongue squamous cell carcinoma via miR-375
}

\author{
LINGFEI JIA ${ }^{1,2^{*}}$, SHAN ZHANG ${ }^{1 *}$, YIPING HUANG ${ }^{3 *}$, YUNFEI ZHENG ${ }^{3}$ and YEHUA GAN ${ }^{1,2}$ \\ ${ }^{1}$ Central Laboratory, Departments of ${ }^{2}$ Oral and Maxillofacial Surgery, and ${ }^{3}$ Orthodontics, \\ Peking University School and Hospital of Stomatology, Beijing 100081, P.R. China
}

Received June 19, 2016; Accepted November 11, 2016

DOI: $10.3892 /$ or.2016.5261

\begin{abstract}
Trichostatin A (TSA), a histone deacetylase inhibitor, is used as an anti-carcinogenic and radiosensitizing agent in various cancers. However, the role and mechanism underlying its radiosensitization of tongue squamous cell carcinoma (TSCC) remains unclear. Thus, in this study we aimed to confirm the promotion of miR-375 expression by TSA, and to investigate the effects of TSA and miR-375 in the radiosensitivity of TSCC cells. The results showed that TSA had significant radiosensitizing effects on TSCC cells and miR-375 overexpression had effects similar to TSA in sensitizing these cells to radiotherapy. By contrast, miR-375 knockdown attenuated apoptosis induced by radiation combined with TSA. Mechanistically, the histone acetylation status of the miR-375 promoter region was increased by TSA, resulting in the upregulation of miR-375, which led to a decline of PDK1 and phosphorylated AKT. Taken together, our data suggest that TSA increases the radiosensitization and apoptosis in TSCC cells at least partially via miR-375, and TSA or miR-375 in combination with radiotherapy may provide a valuable approach for the treatment of TSCC.
\end{abstract}

\section{Introduction}

Tongue squamous cell carcinoma (TSCC) is the most common type of oral cancer and is well-known for its high rate of proliferation and nodal metastasis (1). Radiotherapy alone or in combination with surgery, chemotherapy, or other targeted therapies, plays a critical role in the treatment of patients with advanced stage TSCC (2). However, TSCC is characterized as moderately resistant to radiotherapy and the outcome of radiotherapy is often not fully satisfactory due to

Correspondence to: Professor Yehua Gan,CentralLaboratory,Peking University School and Hospital of Stomatology, 22 Zhongguancun Avenue South, Haidian, Beijing 100081, P.R. China

E-mail: kqyehuagan@bjmu.edu.cn

${ }^{*}$ Contributed equally

Key words: tongue squamous cell carcinoma, miR-375, trichostatin A, radiosensitization radioresistance (3). The mechanism of TSCC radioresistance and the way to increase its radiosensitivity still need to be explored.

There have been several studies on the application of histone deacetylase (HDAC) inhibitors as anti-carcinogenic and radiosensitizing agents (4-6). Trichostatin A (TSA), a pan-HDAC inhibitor, has been shown to enhance the radiosensitivity of a panel of human carcinoma cells (7-13). However, its effect on TSCC cell radioresistance and the underlying regulatory signal transduction pathways remain unclear.

Accumulating data have demonstrated that miRNAs, such as miR-23a (14), miR-34b (15), miR-153 (16), and miR-200c (17), play important roles in tumor radiosensitivity by regulating DNA damage repair, cell-cycle arrest, apoptosis, radiation-related signaling, and the tumor microenvironment (18). Moreover, it has been reported that epigenetic modulation such as histone deacetylation contributes to the silencing of miR-375 (19), while TSA restores its expression (20). Furthermore, our previous study demonstrated that miR-375 has antitumor effects in TSCC (21). This led us to speculate that miR-375 is involved in the pharmacological regulation of radioresistance by TSA.

Protein kinase B (AKT or PKB) is one of the major intermediary molecules in the phosphatidylinositol-3-kinase signaling pathway. It plays a crucial role in essential cellular functions and contributes to tumorigenesis and tumor metastasis (22). Previous studies have shown the important role of AKT signaling pathway in the radioresistance of cancer cells $(3,23,24)$. It has been demonstrated that miR-375 inhibits 3 -phosphoinositide-dependent protein kinase 1 (PDK1) and subsequently affects the AKT pathway (25-27). Thus, we further hypothesized that TSA increases the radiosensitivity of TSCC by miR-375 -mediated suppression of AKT pathway.

In this study, we aimed to confirm the effects of TSA on the radiosensitivity of TSCC cells, and to determine whether the miR-375 mediates the pharmacological regulation of radioresistance by TSA.

\section{Materials and methods}

Cell culture and treatments. The human tongue cancer cell lines SCC-15 and CAL27 were purchased from the American Type Culture Collection (ATCC, Manassas, VA, USA) and cultured in Dulbecco's modified Eagle's medium (DMEM) 
supplemented with $10 \%$ fetal bovine serum (FBS) and $1 \%$ antibiotics at $37^{\circ} \mathrm{C}$ in a humidified atmosphere of $5 \% \mathrm{CO}_{2}$ and 95\% air. Cells were irradiated using the HL-2000 HybriLinker (UVP, Upland, CA, USA) at a wavelength of $254 \mathrm{~nm}$. TSA was from Sigma-Aldrich (St. Louis, MO, USA), dissolved in dimethyl sulfoxide and stored at $-80^{\circ} \mathrm{C}$.

RNA oligoribonucleotides. The chemically-modified doublestranded miR-375 mimic and the corresponding miRNA mimic control; a chemically-modified single-stranded miR-375 inhibitor and the corresponding miRNA inhibitor control were from RiboBio Co. (Guangzhou, China). The miR-375 mimic sequences were as follows: sense, 5'-UUU GUU CGU UCG GCU CGC GUG A-3', and antisense, 5'-AAA CAA GCA AGC CGA GCG CAC U-3'; the miRNA mimic control sequences were: sense, 5'-UUU GUA CUA CAC AAA AGU ACU G-3', and antisense, 5'-AAA CAU GAU GUG UUU UCA UGA C-3'; the miR-375 inhibitor sequence was: 5'-UCA CGC GAG CCG AAC GAA CAA A-3'; and the miRNA inhibitor control sequence was: 5'-UCU ACU CUU UCU AGG AGG UUG UGA-3'.

Transient transfection. The cells were plated onto 6-well plates before transfection. After reaching $80 \%$ confluence, the cells were transfected with $30 \mathrm{nM}$ miRNA mimic or $100 \mathrm{nM}$ miRNA inhibitor using Lipofectamine 2000 (Invitrogen, Carlsbad, CA, USA) according to the manufacturer's instructions.

RNA isolation and quantitative reverse-transcription polymerase chain reaction ( $q R T-P C R)$. Total RNA was extracted using the TRIzol reagent (Invitrogen) then reverse-transcribed into complementary DNA using a High Capacity cDNA Reverse Transcription kit (Applied Biosystems, Foster City, CA, USA). Quantitative PCR was conducted at $95^{\circ} \mathrm{C}$ for $10 \mathrm{~min}$ followed by 40 cycles of $95^{\circ} \mathrm{C}$ for $15 \mathrm{sec}$ and $60^{\circ} \mathrm{C}$ for 1 min using the ABI PRISM 7500 real-time PCR system (Applied Biosystems). The primer used for miR-375 reverse transcription was as follows: GTC GTA TCC AGT GCA GGG TCC GAG GTA TTC GCA CTG GAT ACG ACT CAC GC; the primers for qRT-PCR of miR-375 were: sense, 5'-GTG CAG GGT CCG AGG T-3', and antisense, 5'-AGC CGT TTG TTC GTT CGG CT-3'; and the primers for qRT-PCR of U6 (internal control for miRNA) were: sense, 5'-CTC GCT TCG GCA GCA CA-3', and antisense, 5'-AAC GCT TCA CGA ATT TGC GT-3'. The data were analyzed using the $2^{-\Delta \Delta C t}$ relative expression quantity as described previously (28).

Western blot analysis. Western blot analysis was performed as described previously (28). Briefly, cells were harvested, washed with PBS, and lysed in RIPA buffer. Proteins were separated by $12 \%$ sodium dodecyl sulfate-polyacrylamide gel electrophoresis and transferred to nitrocellulose membranes. Primary antibodies against phosphorylated AKT (p-AKT, Cell Signaling Technology, Beverly, MA, USA), AKT (Cell Signaling Technology), PDK1 (Cell Signaling Technology), and $\beta$-actin (Cell Signaling Technology) were diluted at 1:1,000. The intensities of the bands were quantified using ImageJ software (http://rsb.info.nih.gov/ij/). The background was subtracted, and the signal of each target band was normalized to that of the $\beta$-actin band.
Cell proliferation assays. Cell proliferation was measured using Cell Counting Kit-8 (CCK-8, Dojindo, Kumamoto, Japan). Briefly, the cells were plated onto 96-well plates ( $2 \times 10^{3}$ cells/well). After treatment with TSA and/or exposure to a specific dose of radiation, CCK-8 (10 $\mu \mathrm{l})$ was added to each well at various time points and incubated at $37^{\circ} \mathrm{C}$ for $3 \mathrm{~h}$. The absorbance at $450 \mathrm{~nm}$ was measured using a microplate spectrophotometer (Bio-Tek Instruments Inc., Winosski, VT, USA).

Colony formation. CAL27 cells $\left(1 \times 10^{3}\right.$ cells/well) seeded in 6-well plates were treated with TSA, and then exposed to the indicated dose of radiation. The cells were cultured for 2-4 weeks, and the colonies were detected by staining with crystal violet $(0.5 \%$ in $20 \%$ ethanol). Next, cells were harvested and measured by a multifunctional micro-plate reader at $546 \mathrm{~nm}$. The relative colony number (relative survival cell number) $=$ OD 546 administration group/OD 546 control group, and the radiation survival curve was drawn.

TUNEL staining. The cells were cultured on coverslips and treated with TSA or transfected with miR-375 mimics. Next, the cells were exposed to a specific dose of radiation. Then the cells were subjected to TUNEL staining (one-step TUNEL Apoptosis Assay kit, Beyotime, Jiangsu, China) according to the manufacturer's protocol. Briefly, cells were fixed in $4 \%$ paraformaldehyde, treated with $0.1 \%$ Triton $\mathrm{X}-100$, and labeled with fluorescein-12-dUTP using terminal deoxynucleotidyl transferase. Cell nuclei were stained with DAPI. All fluorescent images were examined using a Leica DM3000 microscope (Leica, Solms, Germany).

Measurement of caspase-3/7 activity. The cellular enzymatic activity of caspase-3 and -7 was determined using a colorimetric assay (Caspase-Glo 3/7 Assay Systems, Promega, Madison, MI, USA). Briefly, for each reaction, cells were lysed and incubated with a luminogenic substrate containing the DEVD sequence, which is cleaved by activated caspase-3/7. After incubation at room temperature for one hour, luminescence was quantified using a Centro XS3 LB 960 luminometer (Berthold, Bad Wildbad, Germany).

Chromatin immunoprecipitation (ChIP) assays. The acetylation of miR-375 promoter was assessed using ChIP, which was performed using an EZ-Magna ChIP assay kit (Merck Millipore, Darmstadt, Germany). Briefly, we sonicated the crosslinked chromatin DNA to generate 200-1,000 bp DNA fragments. DNA-protein complexes were immunoprecipitated with specific antibodies against acetyl-histone H3 (ac-H3). Normal IgG was used as the negative control. The protein/ DNA complexes were then eluted and reverse cross-linked. Spin columns were used to purify the DNA. The precipitated DNA was quantified by qPCR. Relative enrichment was calculated as the amount of amplified DNA normalized to the input and relative to values obtained after normal IgG immunoprecipitation. The primer specific for human GAPDH was used as positive control. The sequences are as follows: sense, 5'-TAC TAG CGG TTT TAC GGG CG-3', and antisense, 5'-TCG AAC AGG AGG AGC AGA GAG CGA-3'. The primer sequences for ChIP-1 are as follows: sense, 5'-ACT ACA TCG CCT GGG 
A
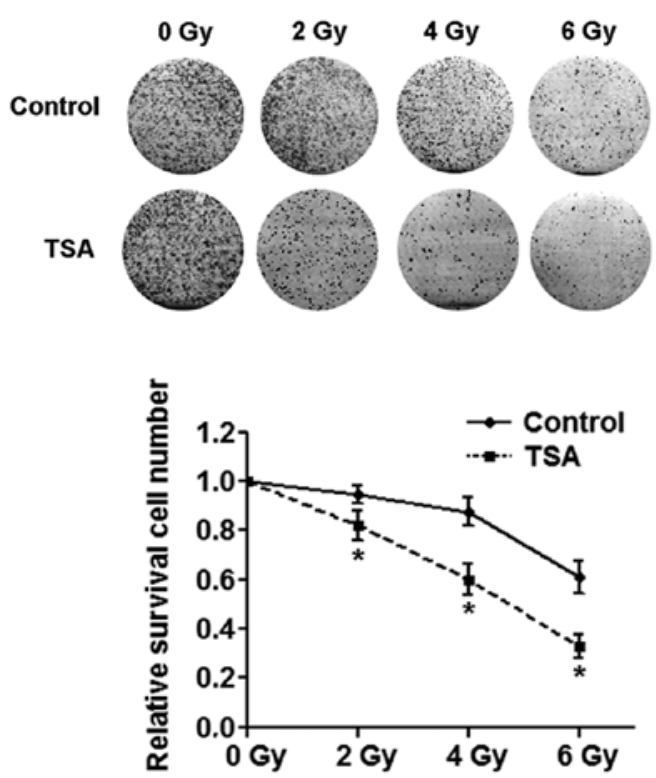

B
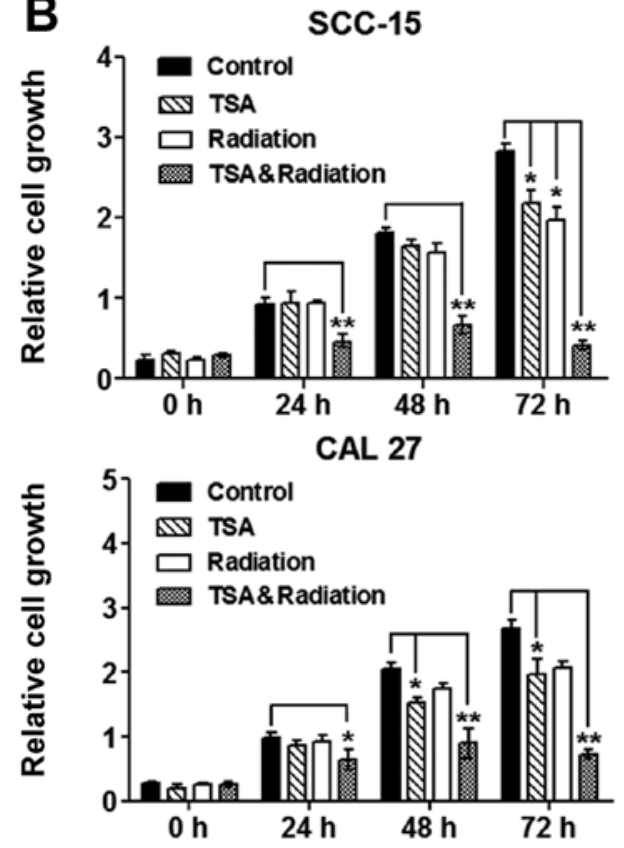

Figure 1. TSA enhances the radiosensitization of TSCC cells. (A) The CAL27 cells were exposed to the indicated dose ( $0,2,4$, or 6 Gy) of irradiation with or without pretreatment of TSA $(1 \mu \mathrm{g} / \mathrm{ml})$ for $16 \mathrm{~h}$. The results of colony formation assay are shown as a typical image or relative survival cell number. (B) CCK- 8 assay showing proliferation in SCC-15 and CAL27 cells treated with TSA ( $1 \mu \mathrm{g} / \mathrm{ml})$ or irradiation (4 Gy) alone, or in combination. In the combined treatment group, the cells were pretreated with TSA $(1 \mu \mathrm{g} / \mathrm{ml})$ for $16 \mathrm{~h}$ and then irradiated with $4 \mathrm{~Gy}$. Data are presented as mean $\pm \mathrm{SD}\left({ }^{*} \mathrm{P}<0.05,{ }^{* *} \mathrm{P}<0.01\right)$.

TTT GA-3', and antisense, 5'-TGC CAA ATA TGC TGC TGG TT-3'; the ChIP-2 sequences are: sense, 5'-CAC CGC CAG TAA AAG CAT CT-3', and antisense, 5'-CCA TCC TTC CCT CTC AGA GC-3'.

Statistical analysis. Statistical analyses were performed using SPSS 16.0 (SPSS, Chicago, IL, USA). The data are expressed as the mean \pm standard deviation (SD) from at least three independent experiments. Differences between groups were analyzed using Student's t-test. In cases of multiple-group testing, one-way analysis of variance (ANOVA) was used. A two-tailed value of $\mathrm{P}<0.05$ was considered statistically significant.

\section{Results}

TSA inhibits TSCC cell proliferation and sensitizes TSCC cells to radiation. To determine the dose of irradiation and TSA that could be used without significantly affecting cell vitality, TSCC cells were treated with different concentrations of TSA or different dose of irradiation. Neither TSA $(1 \mu \mathrm{g} / \mathrm{ml})$ nor irradiation (4 Gy) significantly affected the vitality of TSCC cells (Fig. 1A), so we used these non-cytotoxic doses in the subsequent experiments. We then used CCK- 8 assays to determine whether TSA can regulate radiosensitivity in TSCC cells. Treatment with TSA or irradiation alone only slightly reduced the cell proliferation until $72 \mathrm{~h}$ after treatment. However, combination treatment with TSA and irradiation significantly reduced the proliferation of SCC-15 and CAL27 cells from 24 to $72 \mathrm{~h}$ after treatment (Fig. 1B). The colony formation ability of CAL27 cells was also examined. The results showed that after a mild dose of irradiation treatment
( $\leq 6 \mathrm{~Gy}$ ), the colony formation of CAL27 cells was gradually attenuated (Fig. 1A), and pretreatment with TSA $(1 \mu \mathrm{g} / \mathrm{ml})$ further enhanced the activity of irradiation (Fig. 1A).

TSA enhances radiation-induced TSCC cell apoptosis. To further confirm that TSA increases the radiosensitivity of TSCC cells, we measured caspase-3/7 and apoptosis after the treatments. The activity of caspase-3/7 was upregulated after treatment with TSA or irradiation alone, and further upregulated after the combinational treatment with TSA and irradiation (Fig. 2A). Consistently, the number of apoptotic cells revealed by TUNEL staining was slightly increased by treatment with TSA or irradiation alone, and was significantly increased by the combinational treatment with TSA and irradiation (Fig. 2B).

TSA induces miR-375 expression by increasing histone acetylation of miR-375 promoter and subsequently reduces AKT phosphorylation. To determine whether miR-375 is involved in the regulation of radiosensitivity by TSA, we first treated the TSCC cells with TSA in different concentrations $(0.5-2 \mu \mathrm{g} / \mathrm{ml})$ and time $(24-72 \mathrm{~h})$, and found that TSA upregulated miR-375 expression dose-dependently (Fig. 3A) and time-dependently (Fig. 3B). Then, we checked the histone acetylation in the miR-375 promoter region by ChIP assays after TSA treatment. Two pairs of primers were designed to analyze the status of ac-H3 in the promoter. We found that TSA significantly increased the levels of ac-H3 in the fragment containing ChIP-1 and ChIP-2 (Fig. 4A), suggesting that restoration of histone acetylation in the promoter region induces miR-375 expression after TSA treatment. Further, we found that the level of AKT phosphorylation was reduced after 
A

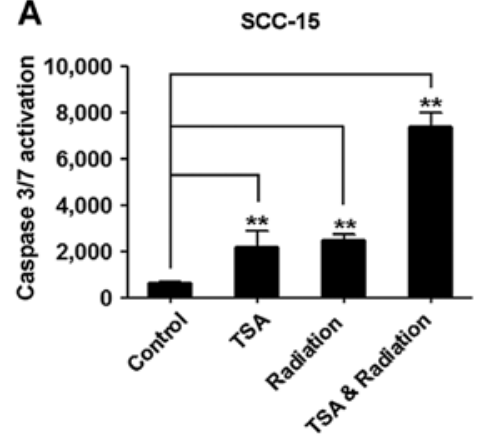

B

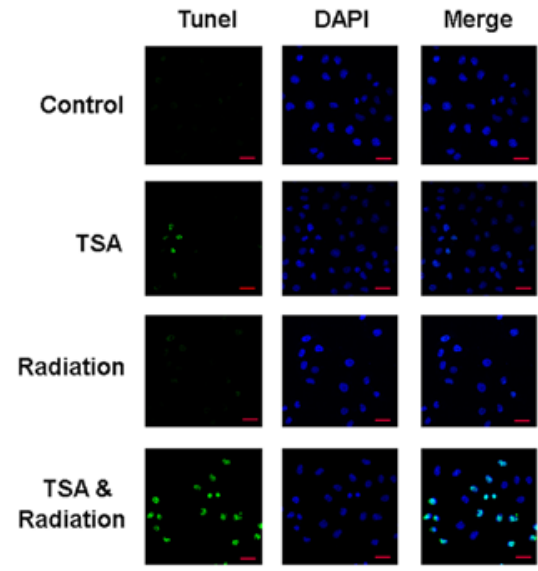

CAL 27
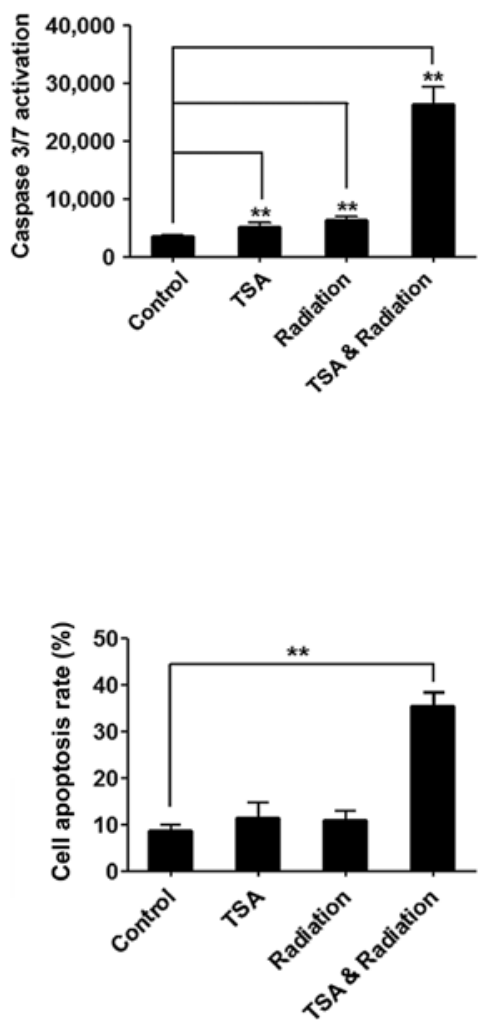

Figure 2. TSA enhances radiation-induced TSCC cell apoptosis. (A) Caspase-3/7 activity in SCC-15 and CAL27 cells treated with TSA (1 $\mu \mathrm{g} / \mathrm{ml})$ or irradiation (4 Gy) alone, or in combination. In the combined treatment group, the TSCC cells were pretreated with TSA $(1 \mu \mathrm{g} / \mathrm{ml})$ for $16 \mathrm{~h}$ and then exposed to $4 \mathrm{~Gy}$ irradiation. (B) Images of TUNEL staining in SCC-15 cells treated as in (A). The cells were labeled with fluorescein-12-dUTP and captured. Scale bars, $100 \mu \mathrm{m}$. Histograms show the percentages of apoptotic cells. Results are presented as the mean $\pm \mathrm{SD}\left({ }^{* *} \mathrm{P}<0.01\right)$.
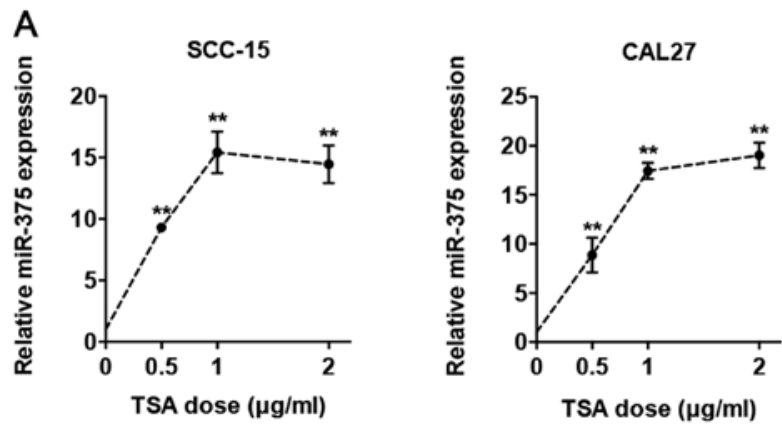

B
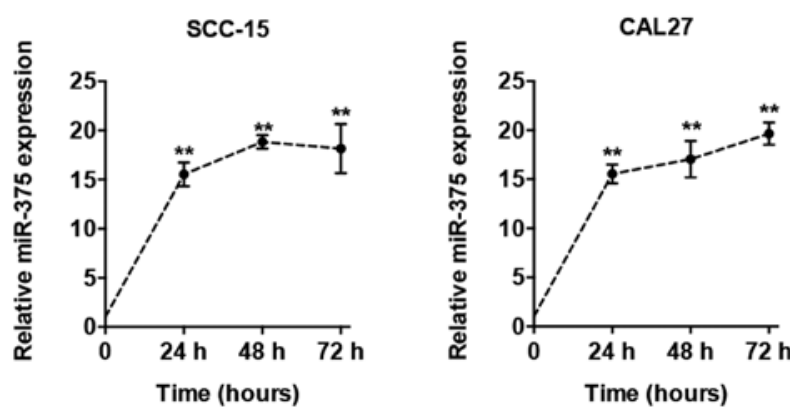

Figure 3. TSA increases miR-375 expression in a dose- and time-dependent manner. (A) Relative miR-375 expression as determined by qRT-PCR in SCC-15 and CAL27 cells with TSA treatment in different doses $(0.5-2 \mu \mathrm{g} / \mathrm{ml})$ for $24 \mathrm{~h}$. (B) Relative miR-375 expression as determined by qRT-PCR in SCC-15 and CAL27 cells with TSA $(1 \mu \mathrm{g} / \mathrm{ml})$ treatment for different times (24-72 h). Data are presented as the mean $\pm \mathrm{SD}\left({ }^{* *} \mathrm{P}<0.01\right)$.
TSA treatment in TSCC cells, and overexpression of miR-375 inhibited the expression of PDK1, followed by the suppression of AKT phosphorylation without significant change of total AKT levels (Fig. 4B).

miR-375 increases TSCC cell radiosensitivity. To determine whether miR-375 overexpression could increase radiosensitivity of TSCC cells, SCC-15 and CAL27 cells were transiently transfected with a control miRNA or miR-375 mimic, and their radiosensitivity was determined. The TSCC cells treated with irradiation or transfected with the miR-375 mimic did not significantly change the percentage of apoptotic cells compared with the control. However, combining overexpression of miR-375 with irradiation induced significant TSCC cell apoptosis compared with the control as revealed by the activity of caspase-3/7 (Fig. 5A) and TUNEL staining (Fig. 5B).

TSA-induced increase of radiosensitivity is partially mediated by miR-375. To determine whether TSA-induced radiosensitivity is mediated by miR-375, an inhibitor specific for miR-375 was used to analyze the effects of miR-375 knockdown on the cellular radioresponse. The inhibition of cell proliferation induced by combining TSA with irradiation was relieved by miR-375 knockdown (Fig. 6A), and the apoptosis induced by the combined treatment was reduced by miR-375 knockdown as revealed by the activity of caspase-3/7 (Fig. 6B) and TUNEL staining (Fig. 6C). 
A

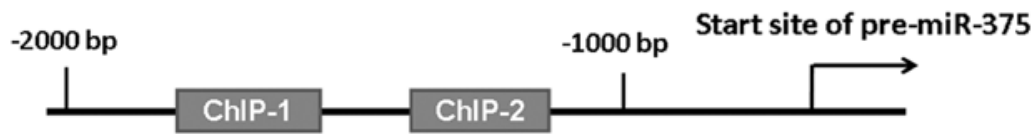

scC-15

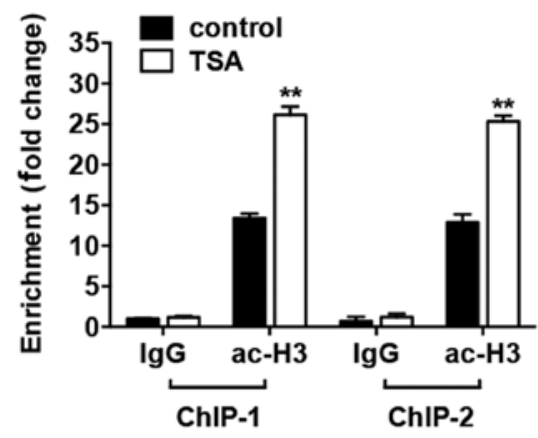

CAL27

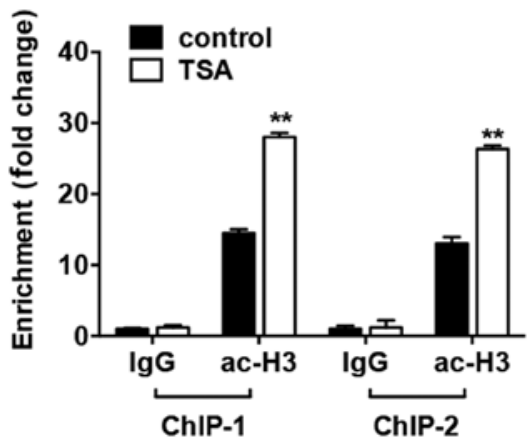

SCC-15

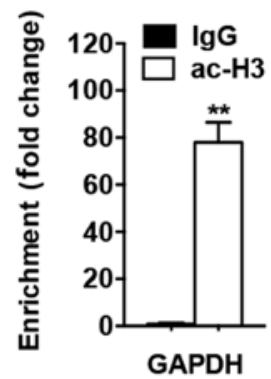

(control primer)

CAL27

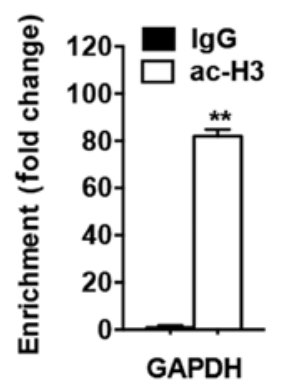

(control primer)

B
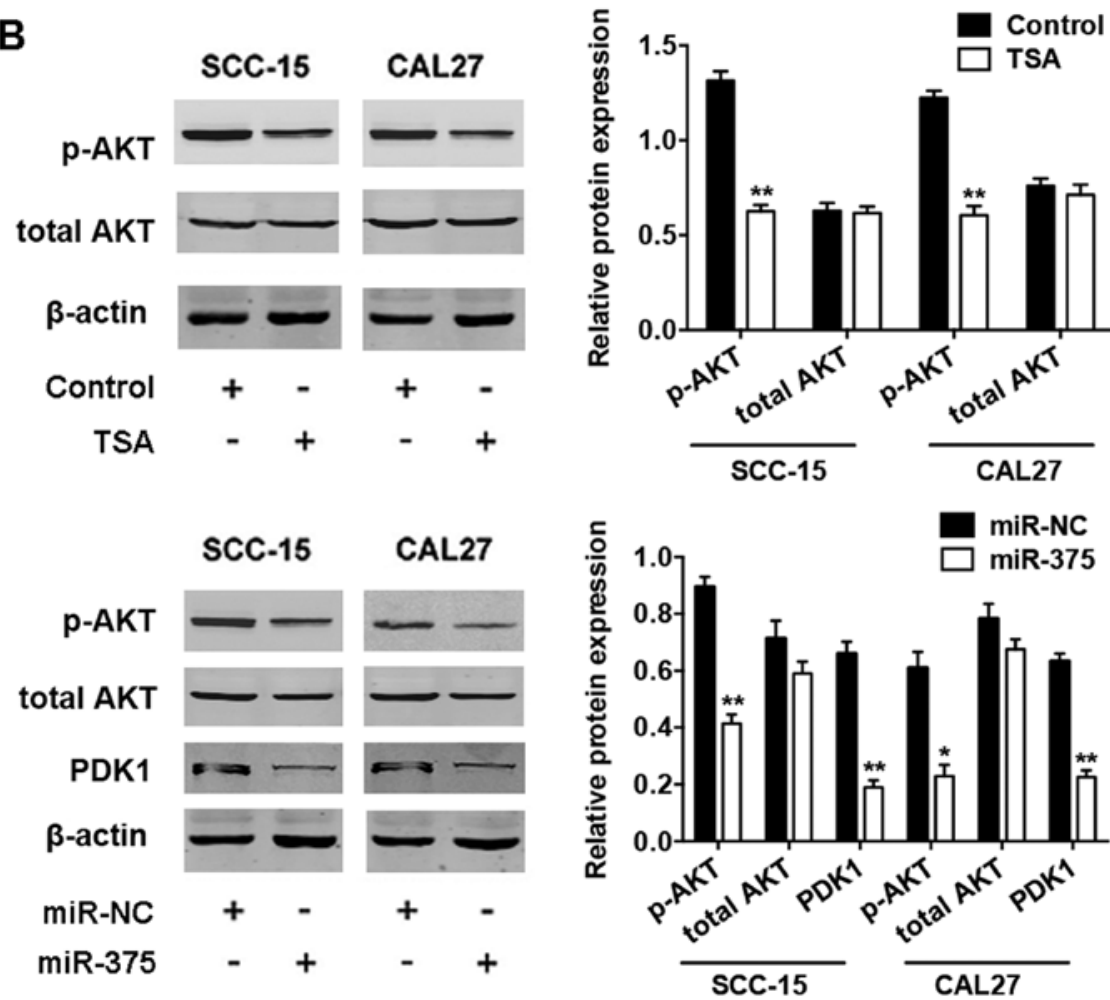

Figure 4. TSA upregulates expression of miR-375 by histone acetylation of the miR-375 promoter and subsequently downregulates phosphorylated AKT expression. (A) Upper, diagram of the miR-375 promoter and location of the primers. Positions marked are relative to the transcriptional start site. Soluble chromatin from SCC-15 (middle) and CAL27 (lower) cells with or without TSA was immunoprecipitated with anti-ac-H3 antibodies, and then immunoprecipitated DNA was analyzed by quantitative real-time PCR. Normal IgG served as negative control. The ac-H3 levels in the promoter region of GAPDH served as positive control. (B) Upper, western blot analysis of protein expression of p-AKT, total AKT, and internal control $\beta$-actin in SCC-15 and CAL27 cells with TSA treatment. Lower, western blot analysis of protein expression of p-AKT, total AKT, PDK1, and $\beta$-actin in SCC-15 and CAL27 cells with miR-375 overexpression. Histograms show the quantification of band intensities. Results are presented as the mean $\pm \mathrm{SD}\left({ }^{*} \mathrm{P}<0.05,{ }^{* *} \mathrm{P}<0.01\right)$. 

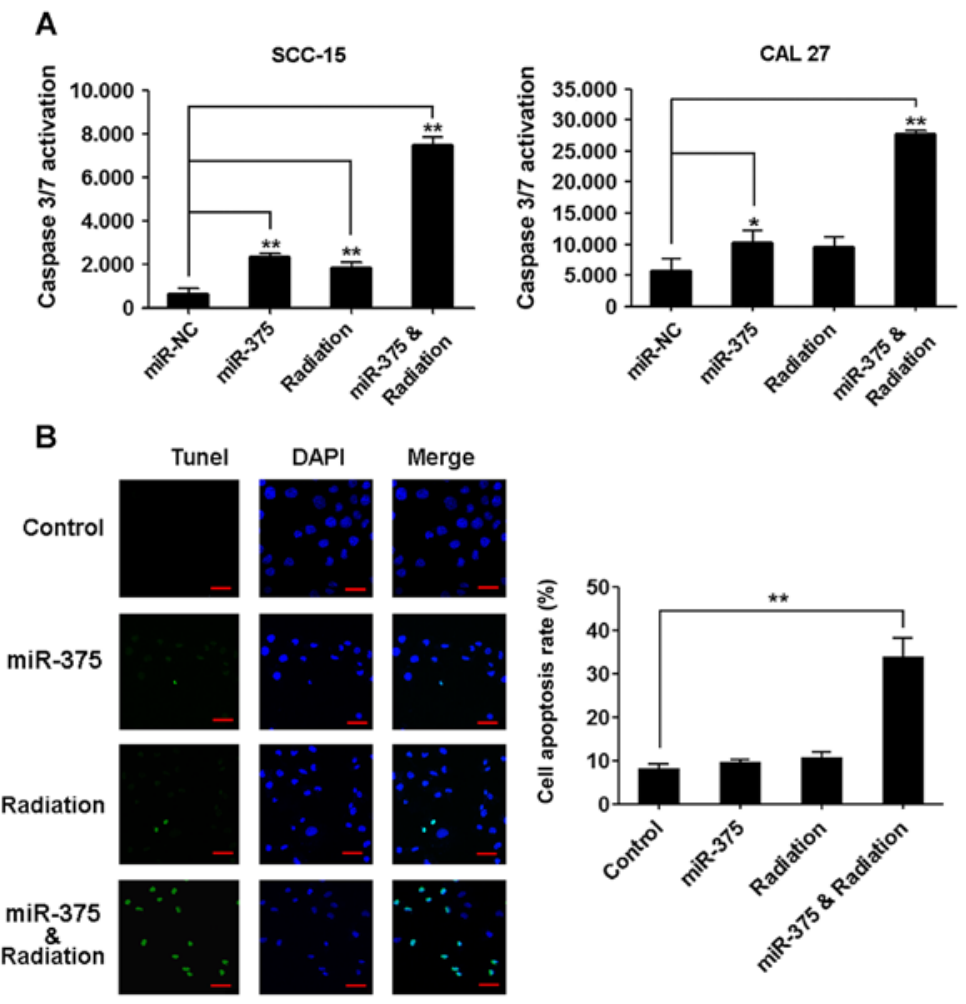

Figure 5. miR-375 increases radiation-induced TSCC cell apoptosis. (A) Caspase-3/7 activity in SCC-15 and CAL27 cells transfected with miR-375 or exposed to irradiation alone, or in combination. In the combined treatment group, the TSCC cells were first transiently transfected with the miR-375 mimic and then exposed to 4 Gy irradiation. (B) Images of TUNEL staining in SCC-15 cells treated as in (A). The cells were labeled with fluorescein-12-dUTP and captured. Scale bars, $100 \mu \mathrm{m}$. Histograms show the percentages of apoptotic cells. Data are presented as the mean $\pm \mathrm{SD}\left({ }^{*} \mathrm{P}<0.05,{ }^{* * *} \mathrm{P}<0.01\right)$.

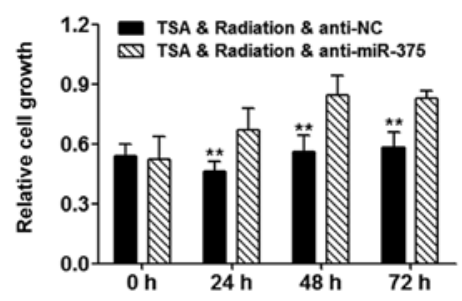

B

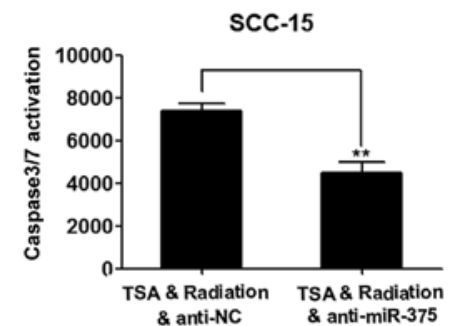

C

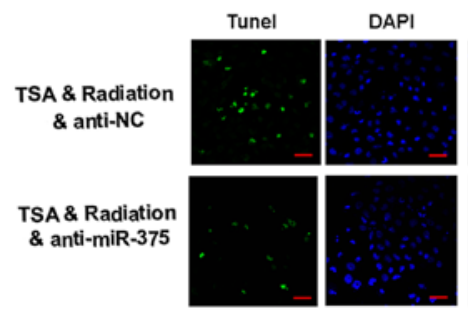

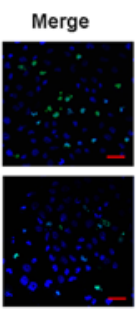

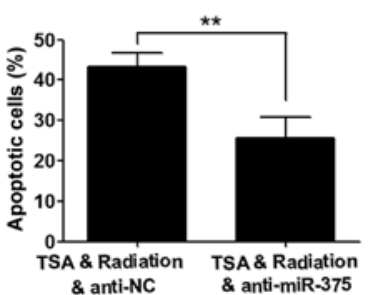

Figure 6. Increased radiosensitivity induced by TSA is reduced by miR-375 knockdown. (A) CCK-8 assays showing proliferation in SCC-15 and CAL27 cells treated with TSA and irradiation, combined with miR-375 knockdown (anti-miR-375) or a non-targeting control (anti-NC). The cells were first transiently transfected with anti-miR-375 or anti-NC, treated with TSA $(1 \mu \mathrm{g} / \mathrm{ml})$ for $16 \mathrm{~h}$, then exposed to 4 Gy irradiation. (B) Caspase-3/7 activity in SCC-15 and CAL27 cells treated as in (A). (C) Images of TUNEL staining in SCC-15 cells treated as in (A), after which the cells were labeled with fluorescein-12-dUTP and captured. Scale bars, $100 \mu \mathrm{m}$. Histograms show the percentages of apoptotic cells. Data are presented as the mean $\pm \mathrm{SD}\left({ }^{* *} \mathrm{P}<0.01\right)$. 


\section{Discussion}

In this study, we demonstrated that TSA sensitized TSCC cells to radiation. TSCC is a common type of cancer from squamous cell carcinomas of head and neck (HNSCCs) and is generally deemed to have intermediate radiosensitivity (3). Our results demonstrated that TSA acted as a powerful radiosensitizer in TSCC cells by inhibiting cell growth and promoting apoptosis, consistent with previous studies in nonsmall cell lung cancer (7), cervical carcinoma (8), colon cancer (9), and erythroleukemic (10). The function of TSA associated with the radiosensitivity of TSCC would advance the development of drugs that increase the sensitivity of TSCC cells to irradiation.

Moreover, we found that TSA increased the histone acetylation of the miR-375 promoter and induced its expression in TSCC cells. miR-375 overexpression increased TSCC cell radiosensitivity, and the radiosensitivity increased by TSA was reduced by knockdown of miR-375, indicating that TSA may at least partially enhance TSCC cell radiosensitivity through miR-375. Regulation of tumor radiosensitivity via miR-associated mechanisms has attracted much attention. Several miRs (14-17) are known to be involved in tumor radioresistance. Previous studies have identified miR-375 as a tumor-suppressor gene in human HNSCC (29-31) and it inhibits cell growth by targeting Sp1 in TSCC (21). However, the exact expression pattern and function of miR-375 in radiosensitive and radioresistant TSCC remains to be explored. In this study, we demonstrated that miR-375 enhanced the radiation-induced TSCC cell apoptosis, which is consistent with a recent study showing that miR-375 promotes the radiosensitivity of cervical cancer cells and increases radiation-induced apoptosis (32). Interestingly, a preclinical study applied liposomal nanoparticles loaded with miRNA in combination with radiotherapy in a lung cancer model and showed that the miRNA delivery plus radiotherapy effectively inhibits tumor growth (33). Thus, miRs-base therapy may be well combined with irradiation to optimize treatment plan in advance.

Furthermore, we found that miR-375 overexpression downregulated PDK1 and phosphorylated AKT expression in TSCC cells, consistent with previous studies demonstrating that miR-375 directly targets PDK1 and subsequently reduces the level of phosphorylated AKT without significant change of total AKT level in pancreatic cells $(25,27)$ and gastric carcinoma (26). AKT pathway, which can be upregulated by radiotherapy, is involved in the mechanisms of radiation resistance $(23,24)$. Especially in HNSCC, the AKT signal transduction pathway is involved in the most important mechanisms of radioresistance: intrinsic radioresistance, tumor-cell proliferation, and hypoxia (3). Several studies have showed that suppressing AKT activity sensitizes cancer cells to irradiation (34-36). Therefore, our study provided evidence that TSA induced apoptosis in TSCC cells by upregulating miR-375 and subsequently suppressing the AKT cascade. However, p53 (32), Sp1 (21), and Notch pathways (37) are also modulated by miR-375, and TSA has also been shown to enhance cellular radiation sensitivity by modulating BRCA1 (11), p53 (12), Bmi-1 (13), hypoxiainducible factor $1-\alpha$, and vascular endothelial growth factor expression (8), all of which are crucial for cell vitality. From these results, we could not exclude the possibility that other signaling pathways are also involved in the regulation of TSCC cell radiosensitivity.

In conclusion, TSA and miR-375 increase TSCC cellular radiosensitivity. TSA enhances radiation-induced TSCC cell apoptosis at least partially by restoring the histone acetylation of the miR-375 promoter and upregulating miR-375 expression, leading to the suppression of PDK1-AKT pathway. Our results suggest that TSA or miR-375, in combination with radiotherapy, may provide a novel approach for the treatment of TSCC.

\section{Acknowledgements}

This study was supported by National Natural Science Foundation of China (81472764 and 81402235), and Foundation of Peking University School and Hospital of Stomatology (PKUSS20140104).

\section{References}

1. Jemal A, Siegel R, Ward E, Murray T, Xu J and Thun MJ: Cancer statistics, 2007. CA Cancer J Clin 57: 43-66, 2007.

2. Calais G, Alfonsi M, Bardet E, Sire C, Germain T, Bergerot P, Rhein B, Tortochaux J, Oudinot P and Bertrand P: Randomized trial of radiation therapy versus concomitant chemotherapy and radiation therapy for advanced-stage oropharynx carcinoma. J Natl Cancer Inst 91: 2081-2086, 1999.

3. Bussink J, van der Kogel AJ and Kaanders JH: Activation of the PI3-K/AKT pathway and implications for radioresistance mechanisms in head and neck cancer. Lancet Oncol 9: 288-296, 2008.

4. Zhu L, Wu K, Ma S and Zhang S: HDAC inhibitors: A new radiosensitizer for non-small-cell lung cancer. Tumori 101: 257-262, 2015.

5. Kim IA, Kim IH, Kim HJ, Chie EK and Kim JS: HDAC inhibitormediated radiosensitization in human carcinoma cells: A general phenomenon? J Radiat Res (Tokyo) 51: 257-263, 2010.

6. Zhang Y, Jung M, Dritschilo A and Jung M: Enhancement of radiation sensitivity of human squamous carcinoma cells by histone deacetylase inhibitors. Radiat Res 161: 667-674, 2004.

7. Zhang F, Zhang T, Teng ZH, Zhang R, Wang JB and Mei QB: Sensitization to gamma-irradiation-induced cell cycle arrest and apoptosis by the histone deacetylase inhibitor trichostatin $\mathrm{A}$ in non-small cell lung cancer (NSCLC) cells. Cancer Biol Ther 8: 823-831, 2009.

8. Yu J, Mi J, Wang Y, Wang A and Tian X: Regulation of radiosensitivity by HDAC inhibitor trichostatin A in the human cervical carcinoma cell line Hela. Eur J Gynaecol Oncol 33: 285-290, 2012.

9. He G, Wang Y, Pang X and Zhang B: Inhibition of autophagy induced by TSA sensitizes colon cancer cell to radiation. Tumour Biol 35: 1003-1011, 2014.

10. Karagiannis TC, Smith AJ and El' Osta A: Radio- and chemosensitization of human erythroleukemic K562 cells by the histone deacetylase inhibitor Trichostatin A. Hell J Nucl Med 7: 184-191, 2004

11. Zhang Y, Carr T, Dimtchev A, Zaer N, Dritschilo A and Jung M: Attenuated DNA damage repair by trichostatin A through BRCA1 suppression. Radiat Res 168: 115-124, 2007.

12. Kim IA, Shin JH, Kim IH, Kim JH, Kim JS, Wu HG, Chie EK, Ha SW, Park CI and Kao GD: Histone deacetylase inhibitor-mediated radiosensitization of human cancer cells: Class differences and the potential influence of p53. Clin Cancer Res 12: 940-949, 2006.

13. Dong Q, Sharma S, Liu H, Chen L, Gu B, Sun X and Wang G: HDAC inhibitors reverse acquired radio resistance of KYSE-150R esophageal carcinoma cells by modulating Bmi-1 expression. Toxicol Lett 224: 121-129, 2014.

14. Qu JQ, Yi HM, Ye X, Li LN, Zhu JF, Xiao T, Yuan L, Li JY, Wang YY, Feng J, et al: MiR-23a sensitizes nasopharyngeal carcinoma to irradiation by targeting IL-8/Stat 3 pathway. Oncotarget 6: 28341-28356, 2015. 
15. Balça-Silva J, Sousa Neves S, Gonçalves AC, Abrantes AM, Casalta-Lopes J, Botelho MF, Sarmento-Ribeiro AB and Silva HC: Effect of miR-34b overexpression on the radiosensitivity of non-small cell lung cancer cell lines. Anticancer Res 32: 1603-1609, 2012.

16. Yang W, Shen Y, Wei J and Liu F: MicroRNA-153/Nrf-2/GPx1 pathway regulates radiosensitivity and stemness of glioma stem cells via reactive oxygen species. Oncotarget 6: 22006-22027, 2015.

17. Shi L, Zhang S, Wu H, Zhang L, Dai X, Hu J, Xue J, Liu T, Liang Y and Wu G: MiR-200c increases the radiosensitivity of non-small-cell lung cancer cell line A549 by targeting VEGFVEGFR2 pathway. PLoS One 8: e78344, 2013.

18. Zhao L, Bode AM, Cao Y and Dong Z: Regulatory mechanisms and clinical perspectives of miRNA in tumor radiosensitivity. Carcinogenesis 33: 2220-2227, 2012.

19. Isozaki Y, Hoshino I, Nohata N, Kinoshita T, Akutsu Y, Hanari N, Mori M, Yoneyama Y, Akanuma N, Takeshita N, et al: Identification of novel molecular targets regulated by tumor suppressive miR-375 induced by histone acetylation in esophageal squamous cell carcinoma. Int J Oncol 41: 985-994, 2012.

20. Yin LH, Zheng XQ, Li HY, Bi LX, Shi YF, Ye AF, Wu JB and Gao SM: Epigenetic deregulated miR-375 contributes to the constitutive activation of JAK2/STAT signaling in myeloproliferative neoplasm. Leuk Res 39: 471-478, 2015.

21. Jia L, Huang Y, Zheng Y, Lyu M, Zhang C, Meng Z, Gan Y and Yu G: miR-375 inhibits cell growth and correlates with clinical outcomes in tongue squamous cell carcinoma. Oncol Rep 33: 2061-2071, 2015

22. Manning BD and Cantley LC: AKT/PKB signaling: Navigating downstream. Cell 129: 1261-1274, 2007.

23. Dent P, Yacoub A, Contessa J, Caron R, Amorino G, Valerie K, Hagan MP, Grant S and Schmidt-Ullrich R: Stress and radiationinduced activation of multiple intracellular signaling pathways. Radiat Res 159: 283-300, 2003.

24. Meng Z and Gan YH: Activating PTEN by COX-2 inhibitors antagonizes radiation-induced AKT activation contributing to radiosensitization. Biochem Biophys Res Commun 460: 198-204, 2015.

25. Hu S, Zhang M, Sun F, Ren L, He X, Hua J and Peng S: miR-375 controls porcine pancreatic stem cell fate by targeting 3-phosphoinositide-dependent protein kinase-1 (Pdk1). Cell Prolif 49: 395-406, 2016

26. Tsukamoto Y, Nakada C, Noguchi T, Tanigawa M, Nguyen LT, Uchida T, Hijiya N, Matsuura K, Fujioka T, Seto M, et al MicroRNA-375 is downregulated in gastric carcinomas and regulates cell survival by targeting PDK1 and 14-3-3zeta. Cancer Res 70: 2339-2349, 2010.
27. Zhou J, Song S, He S, Zhu X, Zhang Y, Yi B, Zhang B, Qin G and Li D: MicroRNA-375 targets PDK1 in pancreatic carcinoma and suppresses cell growth through the Akt signaling pathway. Int J Mol Med 33: 950-956, 2014.

28. Jia LF, Wei SB, Gong K, Gan YH and Yu GY: Prognostic implications of micoRNA miR-195 expression in human tongue squamous cell carcinoma. PLoS One 8: e56634, 2013.

29. Harris T, Jimenez L, Kawachi N, Fan JB, Chen J, Belbin T, Ramnauth A, Loudig O, Keller CE, Smith R, et al: Low-leve expression of miR-375 correlates with poor outcome and metastasis while altering the invasive properties of head and neck squamous cell carcinomas. Am J Pathol 180: 917-928, 2012.

30. Nohata N, Hanazawa T, Kikkawa N, Mutallip M, Sakurai D, Fujimura L, Kawakami K, Chiyomaru T, Yoshino H, Enokida H, et al: Tumor suppressive microRNA-375 regulates oncogene AEG-1/MTDH in head and neck squamous cell carcinoma (HNSCC). J Hum Genet 56: 595-601, 2011.

31. Jamali Z, Asl Aminabadi N, Attaran R, Pournagiazar F, Ghertasi Oskouei S and Ahmadpour F: MicroRNAs as prognostic molecular signatures in human head and neck squamous cell carcinoma: A systematic review and meta-analysis. Oral Oncol 51: 321-331, 2015.

32. Song L, Liu S, Zeng S, Zhang L and Li X: miR-375 modulates radiosensitivity of HR-HPV-positive cervical cancer cells by targeting UBE3A through the p53 pathway. Med Sci Monit 21: 2210-2217, 2015

33. Cortez MA, Valdecanas D, Niknam S, Peltier HJ, Diao L, Giri U, Komaki R, Calin GA, Gomez DR, Chang JY, et al: In vivo delivery of miR-34a sensitizes lung tumors to radiation through RAD51 regulation. Mol Ther Nucleic Acids 4: e270, 2015.

34. Kraus AC, Ferber I, Bachmann SO, Specht H, Wimmel A, Gross MW, Schlegel J, Suske G and Schuermann M: In vitro chemo- and radio-resistance in small cell lung cancer correlates with cell adhesion and constitutive activation of AKT and MAP kinase pathways. Oncogene 21: 8683-8695, 2002.

35. Li X, Chen F, Zhu Q, Ding B, Zhong Q, Huang K, Jiang X, Wang Z, Yin C, Zhu Y, et al: Gli-1/PI3K/AKT/NF-kB pathway mediates resistance to radiation and is a target for reversion of responses in refractory acute myeloid leukemia cells. Oncotarget 7: 33004 33015, 2016.

36. Luo XM, Xu B, Zhou ML, Bao YY, Zhou SH, Fan J and Lu ZJ: Co-inhibition of GLUT-1 expression and the PI3K/Akt signaling pathway to enhance the radiosensitivity of laryngeal carcinoma xenografts in vivo. PLoS One 10: e0143306, 2015.

37. Wang L, Song G, Liu M, Chen B, Chen Y, Shen Y, Zhu J and Zhou X: MicroRNA-375 overexpression influences P19 cell proliferation, apoptosis and differentiation through the Notch signaling pathway. Int J Mol Med 37: 47-55, 2016. 